\title{
First detection of a weak magnetic field on the giant Arcturus: remnants of a solar dynamo?
}

\author{
C. Sennhauser ${ }^{1,2}$ and S. V. Berdyugina ${ }^{2}$ \\ ${ }^{1}$ Institute for Astronomy, ETH Zurich, 8093 Zurich, Switzerland \\ e-mail: csennhau@kis.uni-freiburg.de \\ 2 Kiepenheuer Institut für Sonnenphysik, 79104 Freiburg, Germany
}

Received 21 July 2010 / Accepted 28 February 2011

\begin{abstract}
Context. Arcturus is the second closest $\mathrm{K}$ giant and among the brightest stars in the sky. It has not been found to have a magnetic field, even though $\mathrm{Ca}$ II $\mathrm{H} \& \mathrm{~K}$ lines as activity indicators imply that Arcturus is magnetically active.

Aims. We measure the mean longitudinal magnetic field strengths and interpret them in terms of an intraseasonal activity modulation. Methods. We apply our new Zeeman component decomposition (ZCD) technique to three single sets of Stokes $I$ and $V$ spectra to measure the longitudinal component of the magnetic field responsible for tiny Zeeman signatures detected in spectral line profiles. Results. For two of the spectra, we report the detection of the Zeeman signature of a weak longitudinal magnetic field of $0.65 \pm 0.26 \mathrm{G}$ and $0.43 \pm 0.16 \mathrm{G}$. The third measurement is less significant, but all the measurements closely reproduce a rotationally modulated activity cycle with four active longitudes.

Conclusions. For the first time, a magnetic field on Arcturus is directly detected. This field can be attributed to a diminishing solartype $\alpha \Omega$-dynamo acting in the deepening convection zone of Arcturus. We demonstrate that our new method ZCD lowers the detection limit of very weak magnetic fields from spectropolarimetric measurements.
\end{abstract}

Key words. stars: individual: Arcturus - stars: magnetic field - stars: late-type

\section{Introduction}

Arcturus $(\alpha \mathrm{Boo})$ is a $\mathrm{K} 1.5 \mathrm{III}$ star ascending the red giant branch. It is the second brightest star in the northern hemisphere, thus has been the subject of many studies. Measurements of the Doppler velocity have found long-period variability on timescales of a few hundred days (Gray \& Brown 2006, and references therein), while its short-term variability has been quantitatively characterized by the identification of individual modes of oscillation (Tarrant et al. 2007). Its granulation properties were measured by the same group, with an estimated timescale of $\sim 0.5 \mathrm{~d}$. Being a single star, its mass is relatively poorly known but comparable to that of the Sun $\left(0.8 \pm 0.3 M_{\odot}\right.$ by Bonnel $\&$ Bell 1993). The debate on the effective temperature (Griffin 1996) has waned, and it is nowadays generally accepted to be $4300 \mathrm{~K}$.

Ayres et al. (2003) concluded that Arcturus may sustain a modest level of magnetic activity responsible for the heating of the coronal structures. The long-term study of $\mathrm{Ca}$ II $\mathrm{H} \& \mathrm{~K}$ lines as classical activity indicators by Brown et al. (2008) reveals a range of variability periods between the years 1984 and 2007, exhibiting an apparent magnetic cycle with an estimated duration of $\lesssim 14$ yr. Owing to the known correlation of magnetic activity with surface temperature observed for the Sun (Gray \& Livingston 1997) and other late-type MS stars (Gray et al. 1996b,a, and references therein), periodic temperature changes of $\approx 20 \mathrm{~K}$ inferred from line-depth ratio variations support this theory. Assuming a rotational period of $730 \mathrm{~d}$ (Peterson et al. 1993), or about 2 yr (Gray \& Brown 2006), with $v \sin i=$ $1.5 \pm 0.3 \mathrm{~km} \mathrm{~s}^{-1}$, the seasonal variability (200-250 days) can be attributed to four active longitudes. Observed periods in $\mathrm{H} \& \mathrm{~K}$ line bisectors can be explained by differential rotation and the latitudinal migration of active regions.

The direct detection (via Zeeman effect) of a magnetic field on Arcturus has therefore been long awaited. The first magnetic field measurement of $2.9 \pm 1.8 \mathrm{G}$ reported by Hubrig et al. (1994) was taken right at the activity maximum, but error bars are too high for an unambiguous detection. We developed a new multi-line analyzing technique for polarized spectra, called Zeeman component decomposition (ZCD). We have been encouraged by its performance for simulated Stokes $I$ and $V$ spectra and its ability to recover very weak longitudinal field strengths whose Zeeman signatures are completely embedded in noise (Sennhauser \& Berdyugina 2010), thus applied ZCD to three datasets taken at the Canada-France-Hawaii telescope (CFHT). We report here the detection of a supposedly varying, very weak magnetic field of about half a Gauss at the photosphere of Arcturus. Section 2 describes our observation and the principles of ZCD used for the data analysis. In Sect. 3, we present our results about the seasonal variability caused by four active longitudes. We discuss possible origins for the magnetic field on Arcturus in Sect. 4, and investigate potential sources of systematic errors responsible for spurious signatures in circular polarization. In Sect. 5, we summarize our conclusions.

\section{Observation and analysis}

The three observations of Arcturus were obtained at the CFHT using ESPaDOnS (Donati et al. 2006) on August 2 2006, 


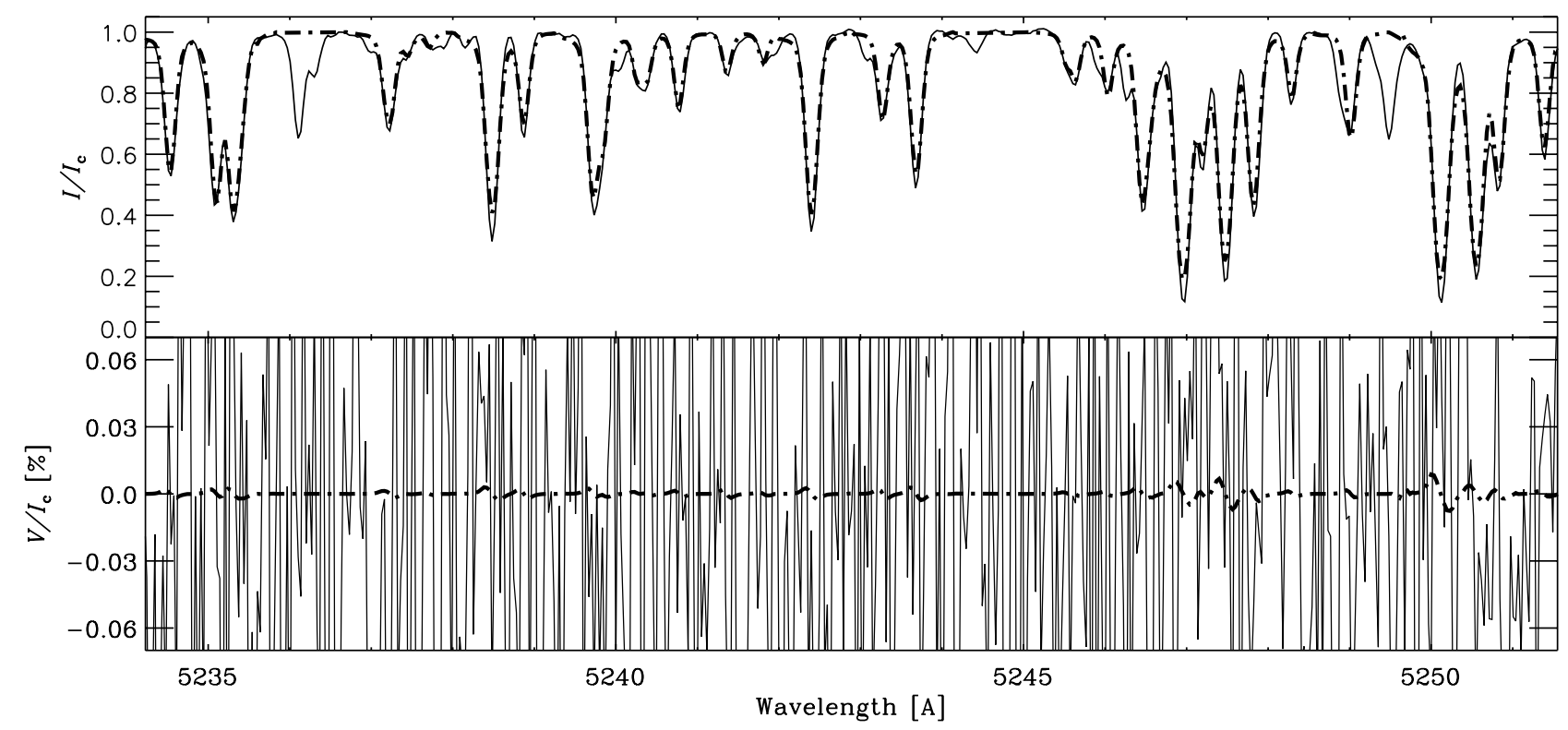

Fig. 1. An interval of Stokes $I, V$ spectra of Arcturus, taken at the CFHT/ESPaDOnS on Aug. 2, 2006 (solid lines), and best fits from ZCD (dash-dotted line). ZCD automatically omits spectral features/lines that cannot be fitted well. Lower panel: the recovered Stokes $V$ signal is fully embedded in noise of the original measurement.

August 23 2008, and December 6 2008. Data reduction was performed using the package Libre-ESpRIT installed at CFHT (Donati et al. 1997). We used ESPaDOnS in circular polarization mode, recording four sub-exposures to obtain the continuumnormalized Stokes $I$ (intensity) and Stokes $V$ (circular polarization) parameters. The total integration times were $16 \mathrm{~s}$ in 2006 and $8 \mathrm{~s}$ in 2008, resulting in a peak signal-to-noise ratio (SNR) well above 1000 in the wavelength region 650-850 nm. However, the SNR gradually decreases toward shorter wavelengths, where most of the spectral lines can be found, dropping below a value of 300 (2006) and 450 (2008) at $\lambda \sim 430 \mathrm{~nm}$.

To recover the mean longitudinal magnetic field, we applied the Zeeman component decomposition analysis (hereafter ZCD), described in Sennhauser \& Berdyugina (2010), which is an inversion technique based on Milne-Eddington assumptions. Treating the strength of each line as a free parameter, ZCD does not rely on pre-calculated line masks, which depend on a given set of stellar parameters. Only the central wavelengths and atomic configurations of the possible lines typically observed for a given spectral class need to be known. During the inversion, ZCD extracts a single line-to-continuum opacity profile common to both Stokes $I$ and $V$ from thousands of lines, and infers a mean longitudinal magnetic field strength at the effective heights of the formation of these lines. In addition, ZCD fits the line strengths to the observations simultaneously for Stokes $I$ and $V$, rejecting lines that cannot be fitted well enough, or whose strengths are below a certain threshold value (usually $1 \%$ residual depth, depending on the type of spectra).

In Fig. 1, we show a fragment of the observations (solid lines) for Stokes $I$ (top panel) and Stokes $V$ (bottom panel). The fitted spectra obtained from ZCD are superimposed as dashdotted lines. We note that the scaling for $V / I_{\mathrm{c}}$ is smaller than the noise level of the observations, to make the fit from ZCD visible.

\subsection{Error estimation}

To estimate the error bars for the recovered magnetic field strength, a Monte Carlo simulation was used for each individual
Table 1. Comparison of recovered $B_{\mathrm{LOS}}$ from LSD and ZCD for two different late-type giants.

\begin{tabular}{cccc}
\hline \hline Name & Spec. type & $\left(B_{\mathrm{LOS}} \pm \sigma\right)_{\mathrm{LSD}}[\mathrm{G}]$ & $\left(B_{\mathrm{LOS}} \pm \sigma\right)_{\mathrm{ZCD}}[\mathrm{G}]$ \\
\hline 32 Cyg & K3Ib + & $1.16 \pm 0.49$ & $0.53 \pm 0.16$ \\
$\lambda$ Vel & K4.5Ib-II & $1.72 \pm 0.33$ & $0.90 \pm 0.13$ \\
\hline
\end{tabular}

spectrum as the most reliable way of error assessment. We followed the Monte Carlo simulation of synthetic data sets described in Press (1992), except that we did not rely on uncertainties for individual pixels of the spectrum. We compute instead standard deviations for equidistant wavelength intervals of the measured Stokes $V$ spectrum and simulated a series of hypothetical data sets (usually 200) with the SNR distribution of the original spectrum. We then applied the ZCD to each of these data sets to obtain 200 values of $B_{\mathrm{LOS}}$. The standard deviation of this distribution gives us an error estimate for the longitudinal magnetic field.

The Stokes $I$ spectrum, which we require to retrieve $B_{\text {LOS }}$ using our ZCD method, remains unchanged. We assume that variations in the net longitudinal magnetic field as small as $\sim 1 \mathrm{G}$ do not influence the intensity spectrum.

\subsection{Analysis of additional spectra}

We previously proved the functionality of ZCD with multiple numerical tests (Sennhauser \& Berdyugina 2010). As a further test, we present results of applying the ZCD to two late-type supergiants 32 Cyg (HD 192909) and $\lambda$ Vel (HD 78647). These stars were previously analyzed by Grunhut et al. (2010), who employed the LSD technique (Donati et al. 1997) and inferred first-order moments of the line-of-sight (LOS) component of the magnetic field. Table 1 compares the results from Grunhut et al. (2010) with the values recovered by our ZCD.

First, we note that whenever there is a magnetic field detection from LSD, there is also one from ZCD (true not only for these two stars). However, while tests have shown that 
the inferred magnetic field strengths from noise-free, simulated spectra of unblended lines are, of course, identical for both methods, the values for these two test objects (exhibiting to some degree balanced antisymmetric Stokes $V$ LSD signatures) from LSD are a factor of two larger than from ZCD. There are several possible reasons for this discrepancy. The mean longitudinal magnetic field $B_{\mathrm{LOS}}$ derived from the LSD Stokes $V$ profile $Z_{V}$ given in velocity space $v$ is proportional to $\int v Z_{V} \mathrm{~d} v$. This quantity is very sensitive to small errors in the far wings of $Z_{V}$, which can be introduced by the following effects:

- The LSD $Z_{V}$ profile is affected by artificial broadening. If one does not account for intrinsic blends (i.e., blends that are not caused by rotational or instrumental broadening), there will be additional broadening of the recovered mean Stokes $V$ profile. This effect strongly depends on the type of spectra and is more severe for late-type stars.

- The wings of $Z_{V}$ are affected by noise, which contributes to the integral more as we go farther into the wings. The choice of integration limits can therefore strongly affect the retrieved $B_{\mathrm{LOS}}$.

- Part of the signature is not caused by the star in question, but by a secondary object (e.g. binary, as for $32 \mathrm{Cyg}$, especially near conjunctions).

One can show quantitatively that broadened, yet otherwise unchanged Stokes $I$ and $V$ profiles result in increasing the magnetic field strength. For instance, the velocity grid $v$, for which the common line patterns $Z_{I}$ and $Z_{V}$ are retrieved, can be replaced with $\tilde{v}=v \cdot s$, where $s$ is a simple scaling (in our case broadening) factor. The nominator $\int \tilde{v} Z_{V} \mathrm{~d} \tilde{v}$ then scales with $s^{2}$, whereas the denominator $\int Z_{I} \mathrm{~d} \tilde{v}$ of the first-order moment only goes as $s$. Therefore, the longitudinal field will also scale with $s$. If we take the intensity profile in Fig. 2 as an example, $s$ is $\sim 1.15$.

The uncertainty introduced by the choice of the measurement window for a noise-free spectrum is about several percent (Kochukhov et al. 2010). This error can be drastically increased by all effects mentioned above, especially in their combination. In addition, known continuum depression of the LSD Stokes I profile and subsequent renormalization also affects the determination of $B_{\mathrm{LOS}}$. All these effects may lead to an overestimate of the magnetic field strength inferred from the first-order moment method for the two example stars.

The uniformity of the magnetic field underlying the current implementation of ZCD is an assumption that may not reflect reality. We conclude that the different assumptions underlying LSD and ZCD may lead to different results. For slowly rotating late-type stars, the more sophisticated treatment of line blending of ZCD, accounting for the different shapes of weak and stronger lines, may render ZCD the more appropriate choice.

\section{Results}

The recovered longitudinal magnetic field strength and corresponding uncertainty values for Arcturus are collected in Table 2. The second column indicates the number of lines used by ZCD. The initial number of lines of over 5000 in our linelist was reduced by the code during inversion, individually for each spectrum, to increase the goodness-of-fit.

Using the average of the retrieved line strengths, the inferred line-to-continuum opacity profile $\kappa_{\mathrm{L}} / \kappa_{\mathrm{c}}$ from ZCD was transformed into mean Stokes $I$ and $V$ profiles, which are presented with solid lines in Fig. 2 for one of the observations. Stokes $I$

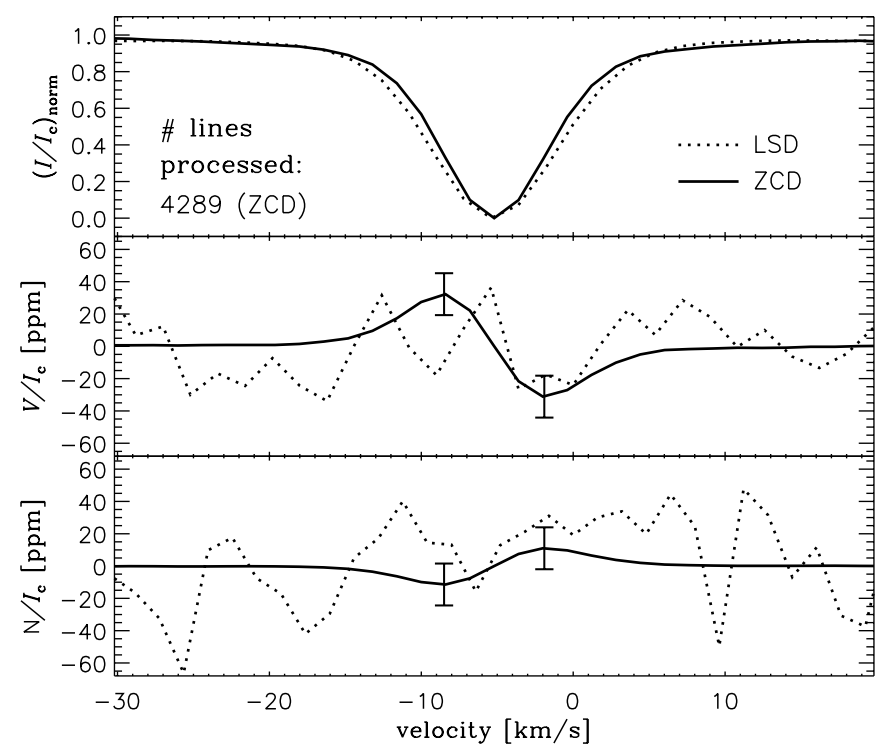

Fig. 2. The recovered ZCD line-to-continuum opacity profile, transformed into a mean Stokes $I$ profile with central depth equal to unity (upper panel), mean Stokes $V$ profile (middle panel), and the diagnostic null spectrum (lower panel), all drawn as solid lines. The error bars were estimated with Monte Carlo simulations. Superimposed as dotted lines are the corresponding mean profiles obtained with the LSD technique.

Table 2. Recovered magnetic field strength for three different spectra (including the diagnostic null spectra, fourth column) of Arcturus.

\begin{tabular}{ccccc}
\hline \hline Obs. date & \# lines & $B_{\mathrm{LOS}}[\mathrm{G}]$ & $B_{\mathrm{LOS}, \text { null }}[\mathrm{G}]$ & $\sigma_{B}[\mathrm{G}]$ \\
\hline 2 Aug. 2006 & 4289 & 0.65 & -0.17 & 0.26 \\
24 Aug. 2008 & 3919 & -0.23 & -0.01 & 0.20 \\
6 Dec. 2008 & 3758 & 0.43 & -0.16 & 0.16 \\
\hline
\end{tabular}

was renormalized to a line central depth equal to 1 . For comparison, the results from the least-square deconvolution (LSD) analysis, described in Donati et al. (1997), were overplotted as dotted lines. In addition, we analyzed a sum of orthogonally polarized spectra, a so-called "null spectrum", which should contain zero circular polarization. We conclude that the influence of blends on the one hand, and the combination of weak and strong lines on the other, caused the LSD profiles to appear slightly broader than the Stokes I signature obtained from ZCD. For Stokes $V$, there is no significant signal in the LSD Zeeman signature, as seen in the middle panel of Fig. 2. However, the Zeeman profile for Stokes $V$ obtained from $\kappa_{\mathrm{L}} / \kappa_{\mathrm{c}}, B_{\mathrm{LOS}}$, for a line at $\lambda=$ $500 \mathrm{~nm}$ and with a Landé factor of 1.23 (the average of all lines processed) shows an amplitude at the $2.6 \sigma$ level, while the profile for the null spectrum is equivalent to zero (lower panel of Fig. 2). We emphasize that these are not mean Zeeman signatures in the terminology of Semel et al. (2009), since ZCD works on the assumptions of a uniform magnetic field. This constraint enables ZCD to lower the detection limit for the mean longitudinal component of magnetic fields.

\subsection{Independent spectral region analysis}

We also wish to demonstrate that an independent analysis of different spectral regions yields compatible values for the LOS magnetic field strength. Although the error bars for the individual subsets are, of course, larger than in Table 2, this 
Table 3. Recovered magnetic field strengths for different spectral regions (including individual error bars and the number of lines processed) of the three Arcturus spectra.

\begin{tabular}{|c|c|c|c|}
\hline $\begin{array}{l}\text { Spectral } \\
\text { interval }[\AA]\end{array}$ & 02/08/06 & $\begin{array}{c}24 / 08 / 08 \\
B_{\mathrm{LOS}} \pm \sigma_{B}[\mathrm{G}] \text { (\# lines) }\end{array}$ & $06 / 12 / 08$ \\
\hline $4000-4300$ & $0.77 \pm 1.00(1067)$ & $0.71 \pm 1.19(1026)$ & $0.60 \pm 0.64(1104)$ \\
\hline $4300-4750$ & $0.61 \pm 0.56(1507)$ & $-0.90 \pm 0.50(1537)$ & $0.73 \pm 0.38(1591)$ \\
\hline $4000-4750$ & $0.59 \pm 0.41(2611)$ & $-0.59 \pm 0.31(2521)$ & $1.01 \pm 0.41(2811)$ \\
\hline $4300-5000$ & $0.54 \pm 0.40(2319)$ & $-0.51 \pm 0.33(2213)$ & $0.43 \pm 0.24(2250)$ \\
\hline $4750-5300$ & $0.79 \pm 0.36(1455)$ & $-0.10 \pm 0.67(1429)$ & $0.32 \pm 0.29(1479)$ \\
\hline $5300-6000$ & $0.59 \pm 0.41(925)$ & $0.04 \pm 0.34$ & $0.31 \pm 0.34$ \\
\hline $5000-6000$ & $0.75 \pm 0.24(1639)$ & $-0.05 \pm 0.24(1655)$ & $0.35 \pm 0.24(1694)$ \\
\hline $6000-7000$ & $0.90 \pm 0.34(674)$ & $0.24 \pm 0.29(550)$ & $0.08 \pm 0.27(583)$ \\
\hline 7000-9000 & $-0.19 \pm 0.72(416)$ & $0.34 \pm 0.67(421)$ & $0.16 \pm 0.37(416)$ \\
\hline
\end{tabular}

analysis provides a good indication that the two magnetic field detections (02/08/2006 and 06/12/2008) are reflected in many parts of the spectrum. In Table 3, we present the determinations of the magnetic field for nine spectral intervals for the three Arcturus spectra. The number of lines processed in each interval is given in parentheses. For larger numbers of lines and longer wavelengths, we expect smaller error bars, which is reproduced well by the Monte Carlo simulation. In this context, the spectral region from 5000 to $6000 \AA$ seems to be the most significant contributor in terms of the magnetic field values inferred from the whole spectrum. Apart from statistical variations, there might be a possible tendency for the magnetic field to decrease at longer wavelengths, as seen in the lower columns for the observations 2 Aug. 2006 and 6 Dec. 2008. Otherwise the detections of a positive magnetic field in observations 1 and 3 are steadily reproduced in the different subsets of the spectra.

\section{Discussion}

As can be seen from Table 2, the recovered photospheric longitudinal magnetic field of Arcturus is perhaps not constant in time. This is indeed expected after taking into account the gaps between the different observations. The $\mathrm{H}+\mathrm{K} S_{\text {II }}$ index observations of Brown et al. (2008) show intraseasonal periods of 253 days between 1984 and 1986 and 207 days in 1986-1989. Trying to see whether our three datapoints are consistent with activity periods of this range, we assumed our strongest value $0.65 \mathrm{G}$ to be the amplitude of an intraseasonal magnetic field variability. While excluding a smaller amplitude, we also assumed that larger values well above $1 \mathrm{G}$ would have been detected by previous studies. Under these assumptions, our three datapoints satisfy a 208-day period, endorsing previous activitycycle measurements. According to the seasonal variability with an estimated minimum cycle duration of $14 \mathrm{yr}$ found by the authors mentioned above, the earliest possible next maximum in $\mathrm{H}+\mathrm{K}$ is 2009 . In this context, our observations appear to have been taken either during a period of increasing activity, or during an extended activity minimum.

\subsection{Possible magnetic field generators}

The first of two possible origins for magnetic fields is a local dynamo from giant convection cells, which is suggested to generate the field of $\sim 1 \mathrm{G}$ on the $\mathrm{M}$ supergiant Betelgeuse, discovered by Aurière et al. (2010).

Owing to the large apparent diameter of Arcturus (21.05 \pm 0.21 mas), Lacour et al. (2008) attempted to directly image its surface via interferometry, using a technique similar to that successfully applied to Altair ( $\alpha$ Aql) by Monnier et al. (2007) with a resolution of $<1$ mas. They failed to detect surface inhomogeneities, and concluded that the most probable brightness distribution is that of a simple limb-darkened disk. However, because of the limited number of resolution elements, the existence of large convection cells, which was modeled by e.g. Kiss et al. (2006), analogous to solar granulation, cannot be ruled out. For Betelgeuse, Haubois et al. (2009) explain their imaging and interferometric observations, and their variability, in terms of these giant granules. These large convective cells could sustain local small-scale dynamos even without stellar rotation (Freytag 2003; Dorch \& Freytag 2003). For Arcturus, however, the predicted number of $\sim 500$ cells, using the scaling relation for the size of stellar granules from Freytag et al. (2002), is too large to explain the power spectrum of the stochastic noise in the data of Brown et al. (2008). In addition, the velocity span of the $\lambda 6252.56 \mathrm{Fe}$ I line lacks any correlation with the predicted 14 yr magnetic cycle. The observed properties mentioned above indicate that this dynamo is not expected to operate on Arcturus, where granules are smaller than on Betelgeuse and display no long-term variability, while magnetic activity is concentrated at active longitudes.

For Arcturus, a solar-like $\alpha \Omega$-dynamo driven by convection and differential rotation is more probable. As outlined by Brown et al. (2008), there is a similarity between the behavior of the Ca II H\&K activity indicator on the Sun and on Arcturus, revealed by line-depth ratio variations with a 2 yr time lag as observed in late-type dwarfs (e.g., Gray 1994; Gray et al. 1996a). In addition to the four suspected active longitudes undergoing latitudinal migration, there are strong indicators that the photospheric and chromospheric characteristics are closely related to the magnetic surface activity of a solar-type MS star. Active longitudes are common among cool, magnetically active stars, ranging in evolutionary stage from zero-MS stars (Berdyugina \& Järvinen 2005) to solar-type stars (Berdyugina \& Usoskin 2003; Lanza et al. 2009) and rapidly rotating red giants (Berdyugina \& Tuominen 1998; Korhonen et al. 2002). Being attributed to a non-axisymmetric component of a largescale magnetic field (Berdyugina et al. 2002; Moss 2005), these longitudes normally coexist with an axisymmetric component responsible for overall cyclic variations in stellar activity. The loss of angular momentum during both the MS and red giant stages diminishes the overall activity and also changes the stellar structure. Modeling shows that a weaker differential rotation supports the dominance of active longitudes, while a stronger differential rotation leads to a dominant axisymmetric mode. 
Thus, active longitudes and a possible 14-yr cycle on Arcturus imply that there is a solar-like dynamo with a weaker differential rotation. The magnetic fields detected in single red giants by Konstantinova-Antova et al. $(2008,2009)$ are likely due to a solar-type dynamo. While all stars in their sample are relatively rapid rotators $\left(9.4<v \sin i<29 \mathrm{~km} \mathrm{~s}^{-1}\right)$, with magnetic fields ranging from 1 to $10 \mathrm{G}$, Arcturus represents one of the two (cf. Aurière et al. 2009) slowly rotating K giant for which a magnetic field possibly generated by a solar-type dynamo has been detected.

\subsection{Sources for circular polarization cross-talk}

When dealing with circular polarization signals at the $10^{-5}$ level, potential sources of spurious Stokes $V$ signals need to be inspected. The first is the possible contamination of circular polarization by other Stokes parameters. For the ESPaDOnS polarimeter, this cross-talk is caused by stress birefringence in both a triplet lens and the atmospheric dispersion corrector (Donati 2006). The ADC and triplet lens used in August 2006 had a linear to circular cross-talk of about $2-3 \%$. Therefore, in principle if linear polarization of $0.1 \%$ was present, then circular polarization of $10^{-5}$ would be the result of a cross-talk.

For example, fractions of linear polarization on the order of $\sim 1 \%$ can appear in absorption lines because of the effect of optical pumping (Happer 1972) in a gas cloud intervening the LOS if the cloud is within about two stellar radii (Kuhn et al. 2007). These linear polarization signals depend on the geometry of the circumstellar environment and can basically take any shape (Kuhn et al. 2010). Since all spectral lines exhibiting more than two magnetic sublevels in their lower state (including hyperfine splitting) may be subject to uneven level populations, hence optical pumping, this can affect the majority of spectral lines in the visible. When converted into Stokes $V$ via cross-talk, the average of all signatures may be misinterpreted as a Zeeman signature. We note that these signatures do not scale according to the Zeeman effect $\lambda^{2} g_{\text {eff }}$ rule, but this dependence cannot be detected at the SNR levels at which we operate.

By invoking this mechanism and a linear-to-circular crosstalk, magnetic fields are not required to explain Stokes $V$ signals of the order of $10^{-4}$ in the case of partially obscured stars (e.g., Herbig Ae/Be or AGB). Since Arcturus has not yet reached the AGB, this model does not apply, but may not be neglected for highly evolved giants, or giants with circumstellar envelopes, for which very weak circular polarization signals are reported (e.g., Aurière et al. 2009, 2010).

Another possible cause of the misinterpretation of Stokes $V$ signals is stellar pulsation. Two subsequent exposures measuring Stokes $I+V$ and $I-V$ by rotating a quarter-wave plate in front of an analyzer can be shifted in wavelength because of a change in radial velocity of the star between the exposures and cause a spurious polarization signal. Hatzes \& Cochran (1993) found day-to-day variations in radial velocities of up to $100 \mathrm{~m} / \mathrm{s}$ for Arcturus. Could this source of cross talk in Stokes $V$ be responsible for the detected Zeeman signatures? At a wavelength of $\lambda=500 \mathrm{~nm}$ and a Landé factor of 1.23 , our half a gauss for the longitudinal magnetic field $B$ corresponds to a Zeeman velocity shift of

$\Delta v_{H}=\frac{e}{4 \pi m} \lambda B \approx 0.35 \mathrm{~m} / \mathrm{s}$.

Assuming a time lag of $1 \mathrm{~min}$ between two subexposures, the upper limit to the radial velocity modulation is $\Delta v_{\text {rad }} \lesssim 0.07 \mathrm{~m} / \mathrm{s}$ (the exposure times themselves being negligibly small, at $4 \mathrm{~s}$ for our observations). This effect is diminished for dual beam polarimeters with beam exchange (e.g., Tinbergen \& Rutten 1997), which are designed to eliminate these systematic errors and sources of spurious signals to first order (Donati et al. 1997). However, a combination of slow polarization modulation and large pulsation amplitudes (or fast convective motions of large cells) can cause spurious signals and should be verified for pulsating stars. If present, these signals would also appear in the diagnostic null spectra, which is not the case here, and can therefore be ruled out.

\section{Conclusion}

For the first time,we have detected a weak magnetic field on Arcturus. The maximum mean longitudinal component measured via Zeeman effect has a strength of $0.65 \pm 0.26 \mathrm{G}$. Our magnetic field measurements endorse an intraseasonal activity variability revealed by other indicators, satisfying a 208-day period. Combined with the observations of Brown et al. (2008), we propose that a diminishing solar-like dynamo is the origin of the magnetic activity, excluding the possibilities of a remnant fossil field as in EK Eri (Dall et al. 2010, and references therein), or the local small-scale dynamos driven by large convection cells, as suggested in the case of the supergiant Betelgeuse (Aurière et al. 2010). The deepening of the convection zone and additional rotational slowdown during the RGB phase may alter the characteristics of an active dynamo mode into a turbulent, or shear-driven $\alpha \Omega$-dynamo acting between the rapidly rotating (helium-)core and the outer shells (Nordhaus et al. 2008). Long-term spectropolarimetric monitoring of Arcturus is needed to confirm its magnetic activity cycle in terms of Zeemandetection, along with the traditional activity indicators.

The performance of the multi-line analysis method ZCD enables us to detect very weak stellar magnetic fields, and makes ZCD a powerful tool for these studies. The present work is the first in a series of analysis of polarimetric measurements of active MS stars, early and late-type giants.

Acknowledgements. We thank Prof. Gregg Wade for providing the two Arcturus Stokes $I V$ spectra recorded in 2008 and for his valuable comments on the paper. This work is supported by the EURYI (European Young Investigator) Award provided by the European Science Foundation (see www. esf.org/euryi http://www.esf.org/euryi) and SNF grant PE002-104552. We acknowledge the use of the VALD atomic database.

\section{References}

Aurière, M., Wade, G. A., Konstantinova-Antova, R., et al. 2009, ApJ, 504, 231 Aurière, M., Donati, J.-F., Konstantinova-Antova, R., et al. 2010, ApJ, 516, L2 Ayres, T. R., Brown, A., \& Happer, G. M. 2003, ApJ, 598, 610

Berdyugina, S. V., \& Tuominen, I. 1998, A\&A, 336, L25

Berdyugina, S. V., \& Usoskin, I. G. 2003, A\&A, 405, 1121

Berdyugina, S. V., \& Järvinen, S. P. 2005, Astron. Nachr., 326, 283

Berdyugina, S. V., Pelt, J., \& Tuominen, I. 2002, A\&A, 394, 505

Bonnel, J. T., \& Bell, R. A. 1993, MNRAS, 264, 334

Brown, S. F., Gray, D. F., \& Baliunas, S. L. 2008, ApJ, 679, 1531

Dall, T. H., Bruntt, H., Stello, D., \& Strassmeier, K. G. 2010, A\&A, 514, A25

Donati, J. F. 2006, www.cfht.hawaii.edu/Instruments/Spectroscopy/ Espadons/

Donati, J.-F., Semel, M., Carter, B. D., Rees, D. E., \& Cameron, A. C. 1997, MNRAS, 291, 658

Donati, J.-F., Catala, C., Landstreet, J. D., \& Petit, P. 2006, in Solar Polarization Workshop 4, ed. R. Casini, \& B. W. Lites, ASP Conf. Ser., 358, 362

Dorch, S. B. F., \& Freytag, B. 2003, in 210th Symp. International Astronomical Union, ed. N. Piskunov, W. W. Weiss, \& D. F. Gray, IAUS, 210, A12

Freytag, B. 2003, in The Future of Cool-Star Astrophysics: 12th Cambridge Workshop on Cool Stars, Stellar Systems, and the Sun, ed. A. Brown, G. M. Harper, \& T. R. Ayres, ASP Conf. Ser., 12, 1024

Freytag, B., Steffen, M., \& Dorch, B. 2002, Astron. Nachr., 323, 213 
A\&A 529, A100 (2011)

Gray, D. F. 1994, Publ. Astron. Soc. Pac., 106, 145

Gray, D. F., \& Brown, K. I. T. 2006, Publ. Astron. Soc. Pac., 118, 1112

Gray, D. F., \& Livingston, W. C. 1997, ApJ, 474, 802

Gray, D. F., Baliunas, S. L., Lockwood, G. W., \& Skiff, B. A. 1996a, ApJ, 465, 945

Gray, D. F., Baliunas, S. L., Lockwood, G. W., \& Skiff, B. A. 1996b, ApJ, 456, 365

Griffin, R. E. M. 1996, Observatory, 116, 404

Grunhut, J. H., Wade, G. A., Hanes, D. A., \& Alecian, E. 2010, MNRAS, 408, 2290

Happer, W. 1972, Rev. Mod. Phys., 44, 169

Hatzes, A. P., \& Cochran, W. D. 1993, ApJ, 413, 339

Haubois, X., Perrin, G., Lacour, S., et al. 2009, A\&A, 508, 923

Hubrig, S., Plachinda, S. I., Hunsch, M., \& Schroder, K. P. 1994, A\&A, 291, 890

Kiss, L. L., Szabó, G. M., \& Bedding, T. R. 2006, MNRAS, 372, 1721

Kochukhov, O., Makaganiuk, V., \& Piskunov, N. 2010, A\&A, 524, A5

Konstantinova-Antova, R., Aurière, M., Iliev, I. K., et al. 2008, A\&A, 480, 475

Konstantinova-Antova, R., Aurière, M., Schröder, K., \& Petit, P. 2009, in IAU Symp., 259, 433
Korhonen, H., Berdyugina, S. V., \& Tuominen, I. 2002, A\&A, 390, 179

Kuhn, J. R., Berdyugina, S. V., Fluri, D. M., Harrington, D. M., \& Stenflo, J. O. 2007, ApJ, 668, L63

Kuhn, J. R., Geiss, B., \& Harrington, D. M. 2010 [arXiv: 1010.0705]

Lacour, S., Meimon, S., Thiébaut, E., et al. 2008, A\&A, 485, 561

Lanza, A. F., Pagano, I., Leto, G., et al. 2009, A\&A, 493, 193

Monnier, J. D., Zhao, M., Pedretti, E., et al. 2007, Science, 317, 342

Moss, D. 2005, A\&A, 432, 249

Nordhaus, J., Busso, M., Wasserburg, G. J., Blackman, E. G., \& Palmerini, S. 2008, ApJ, 684, L29

Peterson, R. C., Dalle Ore, C. M., \& Kurucz, R. L. 1993, ApJ, 404, 333

Press, W. H. 1992, Numerical Recipes (New York: Cambridge University Press) Semel, M., Ramírez Vélez, J. C., Martínez González, M. J., et al. 2009, A\&A, 504, 1003

Sennhauser, C., \& Berdyugina, S. V. 2010, A\&A, 522, A57

Tarrant, N. J., Chaplin, W. J., Elsworth, Y., Spreckley, S. A., \& Stevens, I. R. 2007, MNRAS, 382, L48

Tinbergen, J., \& Rutten, R. 1997, www.ing.iac.es/Astronomy/observing/ manuals/ 